\title{
Research on the Cultivation Strategies of College Students' Physical Exercise Habits in Tianjin
}

\author{
Zhao Huibo \\ Tianjin Agricultural University, Department of Physical Education \\ Tianjin 300384, China
}

\begin{abstract}
The cultivation of physical exercise habits for college students, the main force in the future social construction, is of great significance to the development of their comprehensive quality. Affected by both the diversification of physical education content and the sports activities, the cultivation of physical exercise habits needs to be based on students' enthusiasm, initiative and autonomy for exercise. Therefore, this paper is committed to study the training strategies of college students' physical exercise habits with the combination of the practice of physical education in colleges and universities.
\end{abstract}

Keywords-Tianjin; Physical Exercise Habits; Cultivation Strategies

\section{INTRODUCTION}

As a platform for developing college students' physical and mental health and promoting students' lifelong sports awareness, college physical education has increased the research on college students' sports interest. It has important theoretical and practical value, and it is also one of the basic components of the current university talent training in China. As a carrier of developing college students' physical and mental health quality, physical education teaching is one of the important contents of physical education in colleges and universities. With the help of physical education, big class, competition and other means, starting from the needs of college students' physical and mental health development, Innovate the goals, evaluation and organization of college physical education classrooms. On the basis of stimulating college students' interest in physical exercise, the development and promotion of college students in sports knowledge, sports skills and health awareness are realized, which lays a solid foundation for better improving the quality of higher education in China.

\section{ANALYSIS OF THE CURRENT SITUATION OF COLLEGE STUDENTS PARTICIPATING IN EXTRACURRICULAR PHYSICAL EXERCISE IN TIANJIN}

Among the college students in Tianjin, $49.9 \%$ of the boys think that extracurricular physical exercise is very important, $40.3 \%$ think it is important, and the positive attitude reaches $90.2 \%$. Only $1.5 \%$ of the boys think that extracurricular physical exercise is not important and $0.4 \%$ think it is not important. . $47.7 \%$ of girls think that extracurricular physical exercise is very important, $42.9 \%$ think it is important, and

2017 TianjinEducation committee scientific research projects

(Project Number: 2016ISZD04). positive attitudes reach $90.6 \%$. Only $1.4 \%$ of girls think that extracurricular physical exercise is not important and $0.4 \%$ think it is not important. Table 8 The attitude of male and female college students participating in extracurricular physical exercise showed that there was no significant difference between the two. The data shows that the majority of ordinary college students in Tianjin participate in extracurricular physical exercise and have a positive attitude and correct understanding. We believe that this is the result of the school sports workers earnestly implementing the various school sports policies and policies of the higher authorities, hard work, education guidance for students, and the role of the news media in promoting the importance of sports. College students are increasingly recognized and valued for their role in physical exercise. "Media research shows that the mass media has an extraordinary impact on people's lifestyles, and has a subtle influence on people's values and lifestyles. In schools, the media learns about sports, appreciates sports, loves sports, and participates in sports. Played a very important role. Especially their understanding of sports, the formation of sports values and the study of physical exercise methods and means."

The survey results of attitudes of college students participating in extracurricular physical exercise showed that the first-year university students thought that extracurricular physical exercise was important and very important, accounting for $92.4 \%$, and neutral and negative attitudes accounted for $7.7 \%$; second-year university students thought that extracurricular physical exercise $92.6 \%$ important and very important, $7.4 \%$ with neutral and negative attitudes; third-year university students considered $90.4 \%$ important and very important for extracurricular physical exercise, $9.6 \%$ with neutral and negative attitudes; University IV Grade students accounted for $86 \%$ of the important and very important extracurricular physical exercise, and $14.1 \%$ of the neutral and negative attitudes. We can see that the proportion of students who have taken a positive attitude towards extracurricular physical activity in the third year of university and fourth grade students has decreased year by year (see Figure 1). Table 9 The results of the chi-square test show that there is no significant difference in the probability of taking two-tailed examinations of extracurricular physical exercise attitudes between the second-year university and the third-year university students, and the attitudes of other different grades participating in extracurricular physical exercise are significantly different. There is a very significant difference 
with the fourth graders. This may be related to changes in university life and learning tasks at all levels. In general, college physical education classes only open to the second year of college, and there are no physical education classes in the upper grades. Some senior students prepare for the postgraduate exams, or focus on computer and English proficiency exams, especially in the fourth year of college, busy writing papers and applying for jobs everywhere, easily overlooking the importance of good health.

\section{ANALysis OF THE REASONS AfFECTING THE COLLEGE STUDENTS' PARTICIPATION IN EXTRACURRICULAR PHYSICAL EXERCISES IN TIANJIN}

The statistics on the reasons that affect the participation of male and female college students in extracurricular physical exercise can be seen from the above. Male college students think that the first reason for participating in extracurricular physical exercise is the lack of sports facilities, the second is the heavy burden of learning, and the third is the poor sports foundation. Female college students believe that the first reason for the participation in extracurricular physical exercise is the heavy burden of study, the second is the lack of equipment in the stadium, and the third is the poor sports foundation. The choice of male and female students differs in the order of the first and second. We analyze that the first is that girls pay more attention to learning, and the other is that the most extracurricular physical exercise programs for girls are badminton, running and aerobics. The requirements are relatively low. The most extracurricular physical exercise programs for boys are basketball, table tennis and football. The requirements for venue equipment are relatively high. Therefore, there are some differences in the ordering, but we can be sure that the heavy burden of learning, the lack of equipment in sports venues and the poor sports foundation are the main reasons that affect college students' extracurricular physical exercise. In the process of implementing quality education, the school education concept will change accordingly, the teaching plan will be continuously adjusted, and the student's learning burden should be reduced. With the continuous development of China's economy, the state's investment in education is increasing, and the investment in sports equipment should also increase. For college students with poor sports foundation, we should pay attention to and pay attention to them. We can help them master the exercise methods through practical measures, and benefit every college student through extracurricular physical exercise.

The top three factors affecting the participation of college students in different grades in extracurricular physical exercise are inadequate sports facilities, heavy learning burden and poor sports foundation. It is worth noting that the first- and third-grade college students ranked the sports field in the first place and ranked the learning burden in the second place. The fourth-grade college students ranked the learning burden in the first place, and ranked the sports field equipment in the second place. It seems that different grades feel different because of the different tasks they face. According to our understanding, the fourth-grade university students have fewer classes per week, but they are busy writing graduation thesis, postgraduate study, foreign language and computer exams.
Therefore, they feel that the first study burden has seriously affected the participation in extracurricular physical exercise. How to guide students to arrange their time reasonably and fully appreciate the benefits of extracurricular physical exercise is also a good job for us.

It can be seen from the above that the natural science majors believe that the first reason for the participation in extracurricular physical exercise is the lack of equipment in the stadium, the second is the heavy burden of learning, and the third is the poor sports foundation. College students of social sciences think that the first reason for participating in extracurricular physical exercise is the heavy burden of study. The second is the lack of equipment in sports venues. The third is the poor sports foundation. The choice of college students of different disciplines is different in the order of the first and second. After we communicated with the students, we learned that there are many extracurricular learning materials arranged by the disciplines of social sciences, and we need to take some time to read or memorize. In fact, learning and physical exercise are not a contradiction. Learning is the main task of college students, but it also requires the combination of work and rest. Through extracurricular physical exercise, we can put in a healthy body and energetic energy to achieve the effect of getting twice the result with half the effort. We should strengthen the promotion of physical exercise methods and functions.

The reason why the students of urban students think that the factors affecting the participation in extracurricular physical exercise are the heavy burden of study, the second is the lack of equipment in the stadium, and the third is the poor sports foundation. The reasons for the influence of college students in rural areas are that the first reason is that the sports facilities are insufficient, the second is the heavy burden of learning, and the third is the poor sports foundation. The choice of college students from different places of origin differs in the order of the first and second. Through observation and contact, we feel that, in general, college students from rural areas are more hard-working and hardworking. They usually study harder. Therefore, when the learning tasks are the same as those of urban students, they do not have a huge burden. Insufficient equipment on the site is the most important factor affecting participation in extracurricular physical exercise. From different angles, we study the reasons that affect college students' participation in extracurricular physical exercise, which is to provide reliable data and theoretical basis for more targeted solutions to these problems.

\section{THE CULTIVATION OF PHYSICAL EDUCATION INTEREST AND THE PROMOTION OF COLLEGE STUDENTS' PHYSICAL AND MENTAL HEALTH DEVELOPMENT}

The cultivation of sports interest as a grasp of college students' physical health identity and conscious participation in physical exercise, in the process of college physical education, the development of college students' sports interest as one of the main contents, by increasing the students' cognitive ability of sports, The development of sports skills and health education further promotes the achievement of the fundamental goal of sports health promotion and promotes the 
development of core literacy of college students. As the main force of future social construction, college students' health awareness is the basic requirement of becoming a qualified builder. The development of physical education classroom can better promote the health awareness of college students and the cultivation of lifelong physical exercise awareness. At the same time, the cultivation of physical exercise interest is conducive to the comprehensive development of the comprehensive quality of college students after they enter the society. The cultivation of college students' sports interest transcends the limitations of pure physical education content and sports evaluation methods. Through the use of physical education classrooms, it helps college students to develop awareness of conscious exercise and healthy exercise, and promotes the practicality and health of higher-level personnel training. consciousness. The importance of physical education in colleges and universities is self-evident. On the basis of developing the physical health of college students, it can enhance the psychological and social adaptability of college students. The cultivation of physical activity interest is a people-oriented, practice-oriented process of diversification and flexibility of education. It is of great significance to enrich the way of physical education in colleges and universities and to innovate the organizational form of physical education in colleges and universities. It can be said that the cultivation of sports interest is a supplement and innovation to the goal of physical education in colleges and universities. This kind of supplement is carried out by means of sports elective courses, sports associations, sports clubs, etc., and provides an effective way to promote the effective development of physical education in colleges and universities. Practical teaching content, motor skills and theoretical knowledge.

\section{FACTORS AFFECTING THE CULTIVATION OF COLLEGE STUDENTS' PHYSICAL EXERCISE HABITS}

Different from the physical education in primary and middle schools, the content of physical education in universities is mainly based on the transfer of sports skills. Through the study of sports skills to strengthen the physical quality of students, in this "sports technology core theory" atmosphere, students only In the physical education class, certain sports skills are mastered. On the basis of this, through the independent exercise technique, the students' motor skills are continuously improved, so that students can obtain great satisfaction, thus expressing students' sports passion and forming a good Physical exercise habits.

Students only have a good emotional experience to enhance their interest in physical exercise. It turns out that the desire to improve students' knowledge and the enjoyment of a happy sports experience in the process of learning will greatly increase their interest in sports and facilitate the development of sports habits. . Therefore, actively encouraging interaction in physical education and increasing teamwork is conducive to enhancing students' emotional experience, which plays a good catalyst for students to participate in sports.

Due to the richness of sports programs, physical education classes are mainly based on technical exercises. Teachers should use flexible teaching methods when teaching, giving students sufficient time to practice, so that students can actively, actively and consciously participate in the teaching process. Get a good sports experience. It is necessary to give full play to the leading role of teachers, and to give full play to the initiative of students, and to strengthen students' physical exercise skills in terms of system and assessment methods, in order to lay a foundation for long-term physical exercise habits. A sports society is a social group that aims at sports or sports activities. Due to the mass and social characteristics of sports culture and the non-professional characteristics of majority participation, members have certain conditions in sports and are combined in a certain way. Each person has a functional division of labor and bears certain Responsibility and tasks. Being in the sports society, college students' feedback on sports in the process of sports participation is quite rich. They are evaluated, guided and encouraged by the like-minded people in sports, which makes them form the habit of participating in sports clubs on time. Cultivation plays a big role in promoting.

According to the survey, many colleges and universities lack sports facilities. Due to the maintenance of school sports facilities, a lot of manpower and resources are needed. In the absence of school sports funds, some school sports facilities are not open to students in their spare time. The student's exercise program is greatly reduced, the enthusiasm of exercise is greatly reduced, and the sports club or physical education content can only be carried out based on this, which greatly limits the students' sports enthusiasm and affects students' sports emotions. The development of exercise habits has a great hindrance. School sports culture system and sports culture atmosphere are the intangible factors that affect students' participation in physical exercise and the formation of physical exercise habits. School sports culture includes sports concepts, thoughts, speeches, organizational forms of sports activities, management systems, and sports. Design and venue facilities, campus sports atmosphere has a subtle role in improving students' sports culture, establishing correct sports culture values and forming good physical exercise habits.

\section{Strategies For the Cultivation of College STUDENTS' PHYSICAL EXERCISE HABITS}

As one of the graspers of the cultivation of higher education talents in China, physical education in colleges and universities is based on the development of college students' physical quality and the cultivation of college students' health awareness and physical exercise habits. Therefore, the advanced concept of physical education in colleges and universities is the basis for the development of college students' physical exercise habits. In the process of college sports reform, the first step is to increase the interpretation and research of physical education classrooms, the leading leaders of physical education teaching and physical education teachers in colleges and universities. We must further study the essence of the simultaneous educational philosophy and change the concept of physical education. On the basis of recognizing the value of physical education in the development of physical and mental health of college students, the development of physical education classrooms in sports classrooms has been comprehensively promoted. Only in-depth research and the establishment of a people-oriented concept of physical 
education can be targeted to determine the goals, specific content, and evaluation methods of physical education classrooms, in order to provide students with the most needed physical education content and organization. The cultivation and development of college students' sports interest is based on the development of lifelong sports concept and social adaptability. It combines lifelong physical education with health education to realize the physical and mental health and comprehensive development of college students, in order to better play the physical education. A solid foundation for the function of nurturing and nurturing. This is also an important trend in the development of college physical education towards scientific and social development.

The cultivation of college students' sports interest pays attention to the implementation of the concept of educating people. In order to better implement the sports interest training of college students, it is necessary to determine the main body of sports work and clarify the goal of college students' sports interest development. The mode of physical education should also be taught from the traditional knowledge and skills, to the development of college students' health consciousness and lifelong sports consciousness. By constructing the modern physical education mode of teacher-led and student subject, the effect of college students' sports interest development is enhanced. . At the same time, college students, as the main body of physical education, should pay attention to the research on the health needs of college students in the process of physical education. On the basis of the sports cognition and practice ability of college students, through the diverse content of physical education, Flexible physical education organization mode, to fully stimulate college students' sports interest, realize the scientific and comprehensive goal of college physical education teaching, make college physical education classroom become a main platform to promote college students' personality improvement, physical and mental health development, and social adaptability .

In the process of cultivating students' sports interest, teachers should implement the principle of educating people, improve the research on sports textbooks according to the basic conditions of college students, and promote the goal of college students' interest in sports through innovative sports teaching organization. Effective physical education teaching methods and organization methods are the core issues to improve the sports interest training of college sports college students, and also a key factor in achieving the goal of college students' sports interest training. Therefore, in the process of college physical education, teachers should mobilize the enthusiasm of college students' physical education with rich and flexible physical education methods, stimulate students' interest in physical education and exercise, cultivate college students' sports skills, and promote college students' physical exercise and lifelong. The development of sports awareness. The diversity of teaching methods requires that each teaching method is targeted. For example, the use of "heuristic" and "inductive" teaching methods is an effective way to stimulate students' interest and cultivate students' sports awareness. At the same time, in the process of college physical education in China, it is necessary to increase the application of information technology in colleges and universities, and promote the improvement of college students' physical and mental health and exercise interest based on the advantages of multimedia technology teaching.

In the process of physical education in colleges and universities, it is necessary to increase the investigation and analysis of the needs of college students' physical education. Based on this, the diversity of college physical education curriculum and the diversification of college students' sports interest training content are realized. Lay a solid foundation with autonomous exercise habits. At the same time, in addition to allowing students to master basic sports knowledge, sports skills, and health care skills in the process of teaching, it is necessary to develop college students' lifelong sports awareness. This needs to encourage and attract college students to participate in sports associations, sports clubs, etc., to improve the path of college students' physical exercise in China, to realize the effective extension and development of college students' sports associations, and to promote the physical education of college students from passive to active. Transformation, from learning to application.

Under the background of rapid changes in sports and college students' sports needs, traditional college physical education teaching can not effectively meet the sports needs of college students. As the leader of physical education in colleges and universities, in order to improve the quality of physical education, it is necessary for the school to continuously build a team of masters to enhance the training effect of college students' sports interest and exercise habits. The construction of the physical education faculty team needs to be based on the existing physical education teachers, give full play to the leading and helping role of excellent physical education teachers, and comprehensively promote the professional skills and theoretical literacy of college physical education teachers through on-the-job training and academic exchanges. , teaching ability, scientific research ability. Therefore, it lays a solid foundation for better promoting the cultivation of college students' physical exercise habits.

\section{CONCLUSIONS}

The cultivation of college students' physical exercise habits is mainly embodied in mastering the core technology of sports, obtaining a good emotional experience of sports and actively participating in sports through the development of sports interests. Based on this, it is a breakthrough, combining physical education with sports associations and integrating schools. Sports resources not only consolidate students' sports skills, but also guide students to transform good sports emotional experience into interest in sports, and instill in lifelong sports health awareness from the perspective of wholehearted development, so that students form good sports motivation. And the need for sports, to maximize the development of students' sports habits.

\section{REFERENCES}

[1] Zhang Kebin. The promotion and encouragement of college physical education to the development of students' sports habits [J]. Journal of Suzhou Education College. 2012. 5( 5)

[2] Mo Lianfang. The definition of college students' physical exercise habits[D]. Shanghai Institute of Metallurgy, Chinese Academy of 
Sciences; Materials Physics and Chemistry (Professional) PhD thesis 2000

[3] You Jiangbo, et al. Research on the effective promotion of physical education habits in physical education teaching $[\mathrm{J}]$. Sports and science. 2009. 9(5)

[4] Long Zhiwei. The influence of the current physical education curriculum model on the development of students' physical exercise habits [J]. Teaching and management. 2001. 1

[5] Wei Zhenbin. Higher vocational physical education should pay attention to the cultivation of college students' physical fitness habits [J]. Private technology. 2011 (6).

[6] Liang Hongxia. Analysis of the factors influencing the cultivation of college students' physical exercise habits [J]. Journal of Neijiang Teachers College. 2008( 4) 